\title{
Using SVPWM direct torque control for permanent magnet synchronous motor
}

\author{
Ao Tang ${ }^{1, a}$, Xiaonan $X^{2, b}$ \\ ${ }^{1}$ School of electrical engineer, Zhengzhou university, Zhengzhou 450001, China \\ ${ }^{2}$ School of electrical engineer, Zhengzhou university, Zhengzhou 450001, China \\ a634910243@qq.com, b195642531@qq.com
}

Keywords: space vector pulse width modulation, Direct Torque Control

\begin{abstract}
The traditional direct torque control belong bang-bang control[1], has a relatively large torque ripple and flux ripple, The space vector pulse width modulation (SVPWM) with direct torque combined[2], can effectively suppress torque ripple and flux, This paper will apply to the feasibility of SVPWM technical in direct torque control[3] system for verification.
\end{abstract}

\section{Introduction}

In recent years, permanent magnet synchronous motor[4] (hereinafter referred to as the PMSM) has aroused more and more attention due to small size, simple structure, light weight, high efficiency, large torque inertia and other advantages. Compared with DC motors, PMSM no commutator and brushes, so low noise and will not produce sparks. Compared with induction motors, PMSM do not need excitation, loss of the stator and rotor parameters can be measured, and therefore power factor is high controllability is good. Compared with the electrically excited synchronous motors, PMSM eliminating the excitation device, simplifying the system so that the volume is reduced, efficiency is improved significantly.And since the advent of direct torque technology, it has been a simple structure, fast dynamic response known, so that the permanent magnet synchronous motor system can achieve a wide range of high-precision positioning control or speed, and good dynamic performance, the permanent magnet synchronous motor applications more widely.

\section{Mathematical Model of PMSM}

PMSM rotor structure by permanent magnets installed in the form of classified into surface-mounted, plug-in and built-in three, this paper will not discussion.

Before creating a mathematical model, first assume that

1) Ignore the stator and rotor reluctance intimate, excluding eddy current loss and reluctance; 2) Ignore core saturation; 3) The permanent magnet material conductivity is zero, the permeability is permeability of vacuum; 4) no damper winding rotor; 5) Excitation magnetic field and the stator field generated by the permanent magnets are sinusoidal not considered harmonics.

In this paper adopt surface-mounted structure, three-phase voltage equation of $\mathrm{ABC}$ coordinates.

$\left(\begin{array}{l}u_{A} \\ u_{B} \\ u_{C}\end{array}\right)=\left(\begin{array}{ccc}R_{s} & 0 & 0 \\ 0 & R_{s} & 0 \\ 0 & 0 & R_{s}\end{array}\right)\left(\begin{array}{l}i_{A} \\ i_{B} \\ i_{C}\end{array}\right)+p\left(\begin{array}{l}\Psi_{A} \\ \Psi_{B} \\ \Psi_{C}\end{array}\right)$

Where $\Psi \mathrm{A}, \Psi \mathrm{B}, \Psi \mathrm{C}$ for the whole flux $\mathrm{A}, \mathrm{B}, \mathrm{C}$ phase windings, $\mathrm{p}$ is the differential operator.

Flux-linkage equation in coordinate of $\mathrm{ABC}$ 
$\left[\begin{array}{l}\psi_{A} \\ \psi_{B} \\ \psi_{C}\end{array}\right]=\left[\begin{array}{ccc}L_{A} & L_{A B} & L_{A C} \\ L_{B A} & L_{B} & L_{B C} \\ L_{C A} & L_{C B} & L_{C}\end{array}\right]\left[\begin{array}{l}i_{A} \\ i_{B} \\ i_{C}\end{array}\right]+\left[\begin{array}{l}\psi_{f A} \\ \psi_{f B} \\ \psi_{f C}\end{array}\right]$

Equation rightmost three were PMG field chain over A, flux B, C windings, and

$L_{A}=L_{B}=L_{C}=L_{\mathrm{s} \sigma}+L_{\mathrm{m} 1}, L_{A B}=L_{B A}=L_{A C}=L_{C A}=L_{B C}=L_{C B}=-\frac{1}{2} L_{\mathrm{m} 1}$

Wherein, $\mathrm{Lm}$ and $\mathrm{Lm} 1$, respectively leakage inductance and magnetizing inductance winding.

Torque equation

$T_{e}=\frac{3}{2} p_{0}\left(\psi_{\alpha} i_{\beta}-\psi_{\beta} i_{\alpha}\right)$

$\psi \alpha, \psi \beta$ and i $\alpha$, i $\beta$ is $\alpha \beta$ coordinate system for the stator flux and current, $p 0$ for the number of pole pairs, Te is the electromagnetic torque, the transformation relations that $\mathrm{ABC}$ system into aßline as following:

$$
\left[\begin{array}{l}
i_{\alpha} \\
i_{\beta}
\end{array}\right]=\sqrt{\frac{2}{3}}\left[\begin{array}{ccc}
1 & -\frac{1}{2} & -\frac{1}{2} \\
0 & -\frac{\sqrt{3}}{2} & -\frac{\sqrt{3}}{2}
\end{array}\right]\left[\begin{array}{l}
i_{A} \\
i_{B} \\
i_{C}
\end{array}\right]
$$

\section{Direct Torque system modeling using the SVPWM}

The main idea of the space vector modulation strategy is in a control period to choose adjacent nonzero-voltage vectors and the zero vector, calculate action time of each vector to synthesize any desired magnitude and phase of voltage vector.Its main task is to: 1) determine the spatial voltage vector where the sector; the role of time 2 ) calculation of the basic voltage vector control; 3 ) calculate the inverter switching action time; 4) to generate PWM wave.

Sector judgment:

$$
\left\{\begin{array}{c}
V_{\mathrm{a}}=V_{s \beta} \\
V_{b}=\sqrt{3} V_{s \alpha}-V_{s \beta} \\
V_{c}=-\sqrt{3} V_{s \alpha}-V_{s \beta}
\end{array}\right.
$$

Order: $\mathrm{a}=1(\mathrm{~A}>0$, opposite $\mathrm{a}=0) ; \mathrm{b}=1(\mathrm{Vb}>0$, contrary $\mathrm{b}=0) ; \mathrm{c}=1(\mathrm{Vc}>0$, contrary $\mathrm{c}=0)$. Reference voltage vector sector where $S=a+2 b+4 c$.

Time:

Now define: $\left\{\begin{array}{c}X=\sqrt{3} V_{s \beta} T_{s} / V_{D C} \\ Y=3\left(\sqrt{3} V_{s \beta} / 3+V_{s \alpha}\right) T_{s} / 2 V_{D C} \\ Z=3\left(\sqrt{3} V_{s \beta} / 3-V_{s \alpha}\right) T_{s} / 2 V_{D C}\end{array}\right.$

Different sectors T1, T2 values by the following table

Table2.1 Relationships of S and T1, T2's

\begin{tabular}{ccccccc}
\hline $\mathrm{S}$ & 1 & 2 & 3 & 4 & 5 & 6 \\
\hline $\mathrm{T}_{1}$ & $\mathrm{Z}$ & $\mathrm{Y}$ & $-\mathrm{Z}$ & $-\mathrm{X}$ & $\mathrm{X}$ & $-\mathrm{Y}$ \\
\hline $\mathrm{T}_{2}$ & $\mathrm{Y}$ & $-\mathrm{X}$ & $\mathrm{X}$ & $\mathrm{Z}$ & $-\mathrm{Y}$ & $-\mathrm{Z}$ \\
\hline
\end{tabular}

Switching time: Sector $\mathrm{S}$ and $\mathrm{cm} 1$, the relationship between $\mathrm{cm} 2, \mathrm{~cm} 3$ in the following table Table 2.2 relationship of $\mathrm{S}$ and $\mathrm{cm} 1, \mathrm{~cm} 2, \mathrm{~cm} 3$

\begin{tabular}{ccccccc}
\hline $\mathrm{S}$ & 1 & 2 & 3 & 4 & 5 & 6 \\
\hline $\mathrm{Cm} 1$ & $\mathrm{~T}_{\mathrm{b}}$ & $\mathrm{T}_{\mathrm{a}}$ & $\mathrm{T}_{\mathrm{a}}$ & $\mathrm{T}_{\mathrm{c}}$ & $\mathrm{T}_{\mathrm{c}}$ & $\mathrm{T}_{\mathrm{b}}$ \\
\hline $\mathrm{Cm} 2$ & $\mathrm{~T}_{\mathrm{a}}$ & $\mathrm{T}_{\mathrm{c}}$ & $\mathrm{T}_{\mathrm{b}}$ & $\mathrm{T}_{\mathrm{b}}$ & $\mathrm{T}_{\mathrm{a}}$ & $\mathrm{T}_{\mathrm{c}}$ \\
\hline $\mathrm{Cm} 3$ & $\mathrm{~T}_{\mathrm{c}}$ & $\mathrm{T}_{\mathrm{b}}$ & $\mathrm{T}_{\mathrm{c}}$ & $\mathrm{T}_{\mathrm{A}}$ & $\mathrm{T}_{\mathrm{b}}$ & $\mathrm{T}_{\mathrm{a}}$ \\
\hline
\end{tabular}


Tables Ta, Tb, Tc are $T_{\mathrm{a}}=\left(T-T_{1}-T_{2}\right) / 4 ; T_{b}=T_{a}+T_{1} / 2 ; T_{c}=T_{b}+T_{2} / 2$

\section{Simulation results and analysis}

Simulation model modules established according to the formulas and tables above, then get integrated permanent magnet synchronous motor using SVPWM direct torque control simulation diagram, as Figure 1 .Set sampling period $\mathrm{T}=0.0001 \mathrm{~s}$ and supply voltage $\mathrm{Udc}=110 \mathrm{~V}$. Motor parameters: inductance $8.5 \mathrm{mH}, \mathrm{Rs}=1.132 \Omega, \mathrm{p} 0=2, \mathrm{~J}=8 \times 10^{-3} \mathrm{~kg} \bullet \mathrm{m} 2$, given flux as $0.3 \mathrm{wb}$, given speed $\mathrm{n}=900 \mathrm{r} / \mathrm{min}$. Give load torque initial to be $2 \mathrm{~N} \cdot \mathrm{M}$, at $0.4 \mathrm{~s}$ to be $5 \mathrm{~N} \cdot \mathrm{M}$.following figures are simulation result .

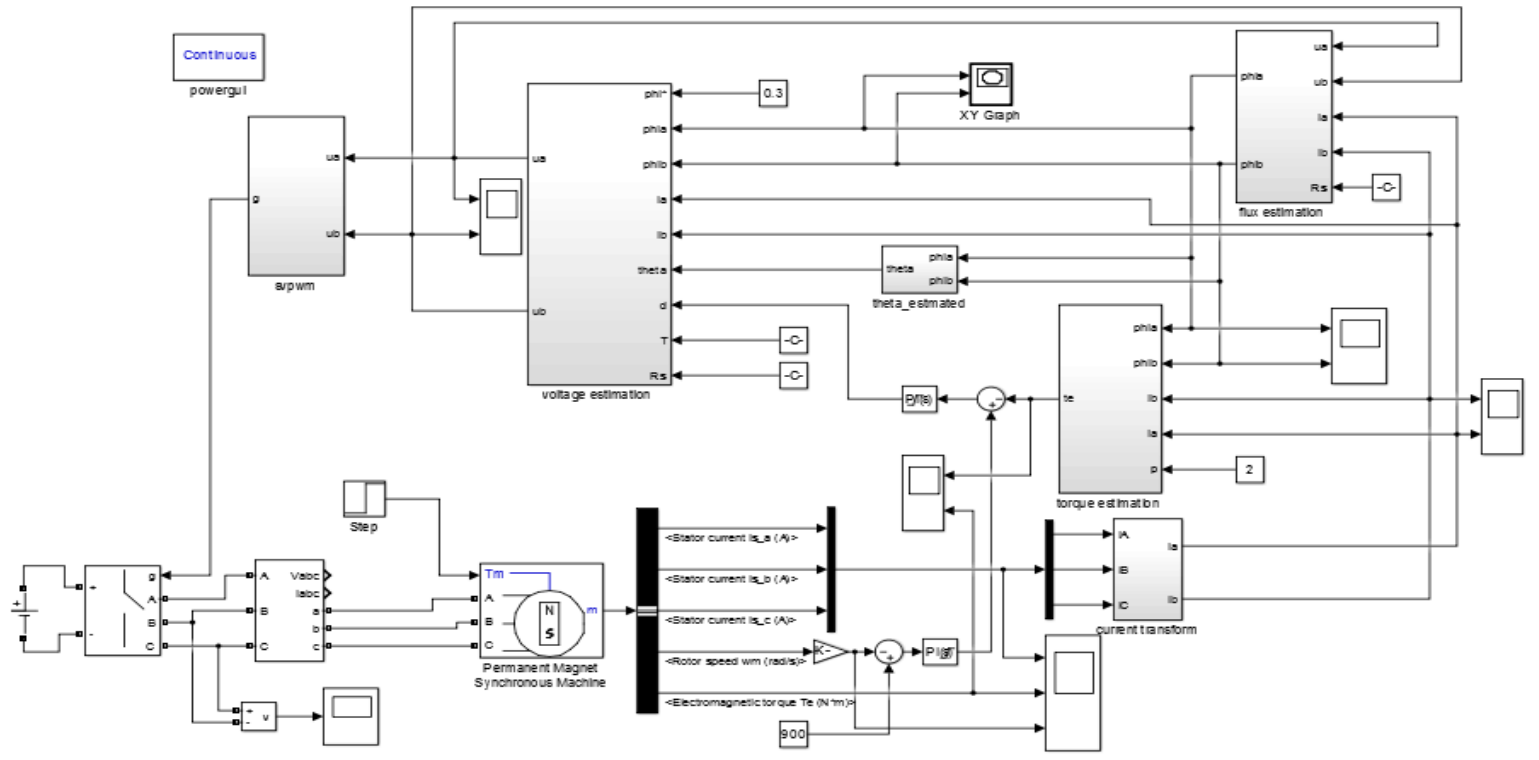

Figure 1 Motor control system simulation diagram

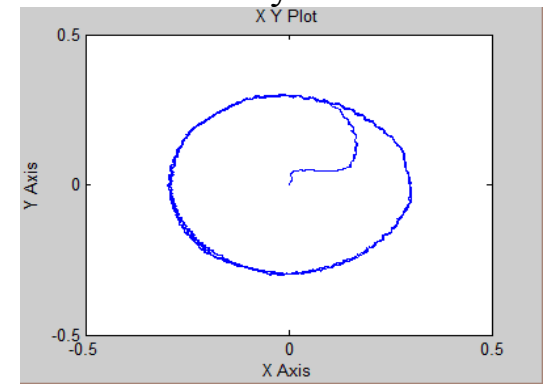

Figure 2 stator flux linkage

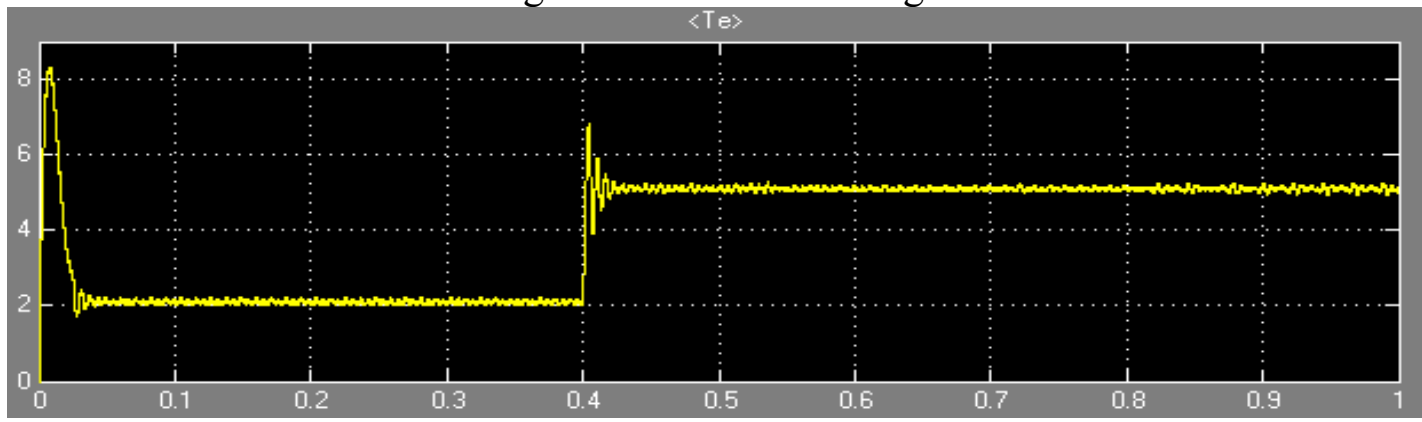

Figure $3 \mathrm{Te}$ 


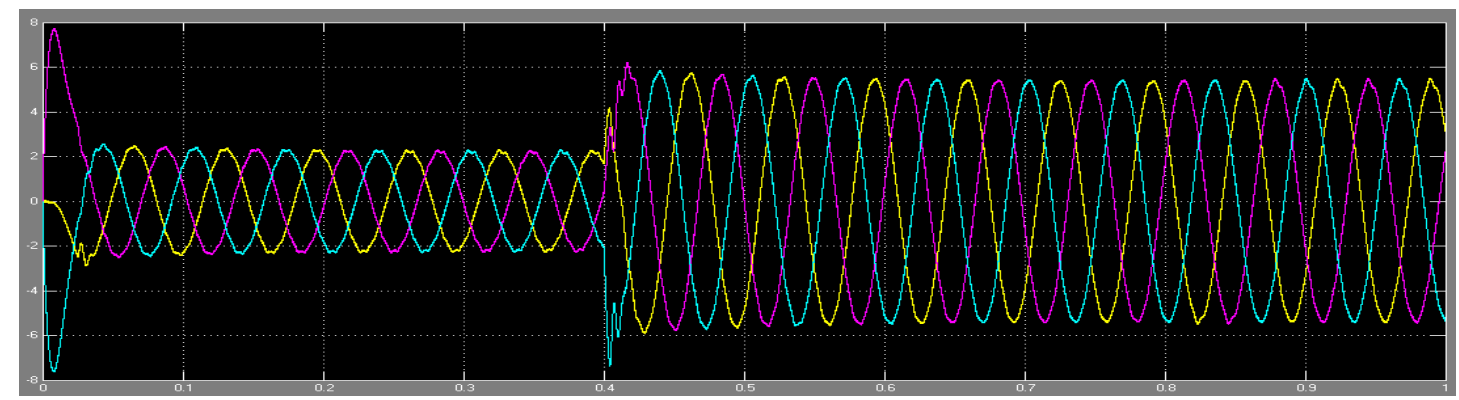

Figure 4 stator three-phase current

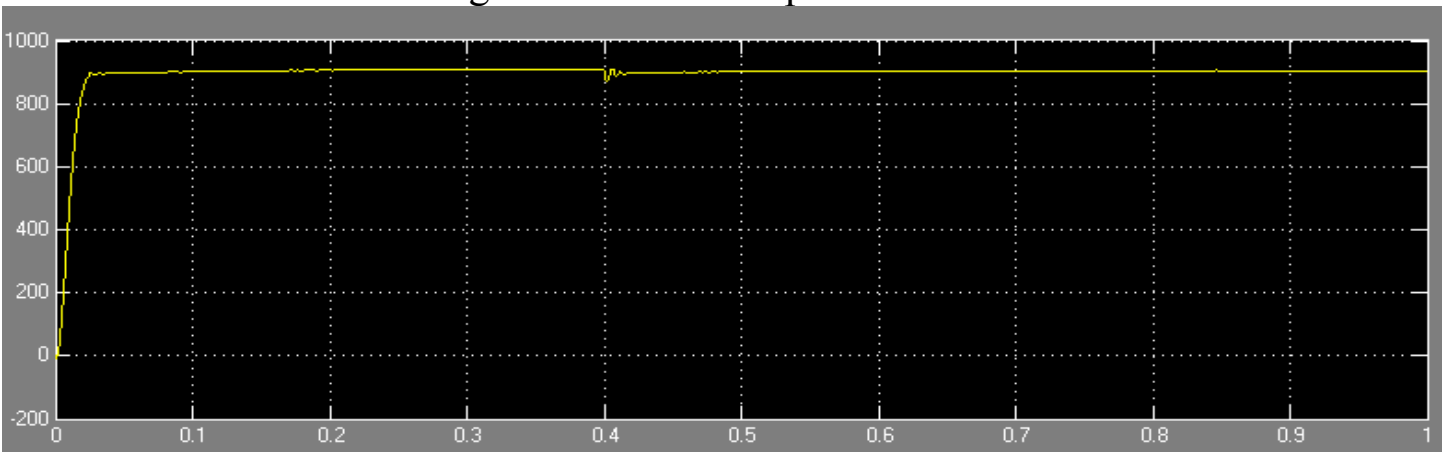

Figure 5 rotate speed

\section{Summary}

According to the results,torque's and speed's response are very fast, there waveforms are smooth and stable. so using SVPWM direct torque control for permanent magnet synchronous motor is viable.

\section{References}

[1]. Depenblock M. Direct self-control(DSC) of inverter-fed induction machine[J] IEEE Trans. on Power Electron, 1988, 3(4): 420-429.

[2]. David Ocen, Luis Romeral, Juan Antonio Ortega, Jordi Cusido,Antoni Garcia. Discrete Space Vector Modulation Applied on a PMSM Motor[C]. 12th International Power Electronics and Motion Control Conference, 2006.8.

[3]. Zhong L, Rahman MF, Hu WY, et al. A direct torque controller for permanent magnet synchronous motor drives[J]. IEEE Trans. on Energy Conversion, 1999, 14(3): 637-642. [4]. Sun Dan, Fang Weizhong, He Yikang. Study on the direct torque control of permanent magnet synchronous motor drives[C]. ICEMS 2001, Shenyang, 2001. 